\title{
Prediction of Power Generation in China Using Process Neural Network
}

\author{
Jianghua Ge*a, Jiwei Wen ${ }^{\mathrm{b}}$, Chuntao Zhang ${ }^{\mathrm{c}}$, Yaping Wang ${ }^{\mathrm{d}}$ and Gang Ding ${ }^{\mathrm{e}}$ \\ School of Mechanical Engineering, Harbin University of Science and \\ Technology, Harbin, Heilongjiang Province, China \\ agejianghua@sina.com, ${ }^{b}$ wenjiweibl@163.com, ${ }^{c}$ zhangchuntaobl@163.com, \\ dwypbl@163.com, edingganghit@163.com
}

\begin{abstract}
The power generation prediction problem can be seen as a time series prediction problem in nature. The traditional time series prediction methods based on regression analysis do not take account into the time accumulation effect existed in the time series due to their discrete input values. This limitation causes the low prediction accuracy of the time series prediction methods based on regression analysis. To solve this problem, a power generation time series prediction model based on the process neural network is proposed. The inputs of the proposed prediction model can be continuous time-varying functions. The time accumulation effect existed in the power generation time series can be expressed and computed by the integration operator of the process neural network. The proposed prediction model is trained and the efficiency of the proposed prediction model is tested by the month power generation data form January 2001 to April 2012, and the comparison experiment results indicate that the process neural network performs better than the auto regression analysis.
\end{abstract}

Keywords: power generation; time series prediction; process neural network; regression analysis; learning algorithm

\section{Introduction}

With the rapid development of the national economy and industry, China's demand for electricity is unprecedented. In order to promote the continuous, fast and harmonious development of national economy, it is necessary to solve the contradictory problem of energy supply and demand, and electric power industry must realize the advance development, it is an important work of the international and domestic electric power planning to predict the power generation ${ }^{[1-3]}$.This can provide decision-making basis for the planning and development of the national grid, and on the other hand it can provide decision-making reference for structural adjustment and development planning of the relevant industry. China's electricity production mainly consists of fire, waterpower, wind and nuclear power, its yield is affected by many complex factors, so it is difficult to establish accurate mathematical model to describe the future trend. In this case, it is possible to predict the power generation by constructing the time series of using accumulative and abundant historical statistics data of power generation, and using the related theory and method of time series prediction.

At present, the common used time series prediction methods are regression analysis and artificial neural network and so on. The related theory of function approximation is the theoretical basis of regression analysis prediction. Because of the constraint of inputting synchronous instant, the input of regression analysis method is a series of discrete values, so it is difficult to fully reflect the accumulation effect actually existed in the time series, and so the prediction 
accuracy remains to be further improved ${ }^{[4,5]}$. The method of artificial neural network prediction is a method of data-driven. It is widely used in time series prediction without special modeling in prior ${ }^{[6-9]}$. In order to solve the problem that regression analysis method is difficult to fully reflect the accumulation effect actually existed in the time series, literature [10] presented a process neural network model that its input is function, literature [11] presented a time series prediction method based on process neural network. A prediction model of power generation based on process neural network is established on the basic of literature [10] and [11], and the power generation of recent years in China is used as the statistical data to apply and test model by using the model.

\section{Time Series Prediction Model based on Process Neural Network}

\subsection{The Process Neuron}

The artificial neuron model is established by imitating biological neuron, and thus the artificial neural network model is established. But the input time accumulated polymerization effect is lacked in traditional artificial neural network. The biological study shows that, outside stimulation for the biological nervous system may last for a period of time, the biological neurons carries out to work in the dynamics principle of information processing according to the received comprehensive . coordination and cumulative effect of multiple time-varying signals in the time delay interval [12]. Therefore, literature [10] presented the process neuron model with the capability of temporal and spatial information processing. The input of process neuron and the corresponding connection weights can be time-varying function or process, adding an operator of time accumulation and aggregation on the basic of traditional artificial neuron aggregation operation of space weighted, making the process neuron also has a capacity of processing space-time information. The process neuron and the traditional artificial neuron is similar in the structure, as shown in figure 1.

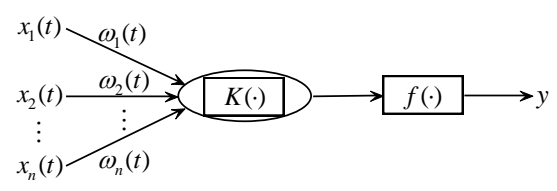

Figure. 1 Process Neuron

The relationship between the output and input to the process neuron:

$$
y=f\left(\sum_{i=1}^{n} \int_{0}^{T} \omega_{i}(t) x_{i}(t) d t-\theta\right)
$$

Among them, $x_{i}(t)(i=1,2, \cdots, n)$ is the input function of process neuron; $[0, T]$ is the sampling interval; $\omega_{i}(t)$ is the corresponding connection weight functions; $\theta$ is the process neuron threshold; $f(\cdot)$ is the activation function, and it can be taken as the Sigmoid function in general.

It is assumed that $x_{i}(t)$ and $\omega_{i}(t)$ are function of $C[0, T]$ in literature [13], however, in the practical engineering, $x_{i}(t)$ and $\omega_{i}(t)$ are always function of $L^{p}[0, T](p>0)$.It is assumed that $x_{i}(t)$ and $\omega_{i}(t)$ are function of $L^{p}[0, T]$ in this paper.

If $x_{i}(t) \in L^{p}[0, T]$, it is knew by extending the Weierstrass approximation theorem in $L^{p}[0, T]$, for $\forall \varepsilon>0$, it must exist polynomial $P(t)$, making $\left|P(t)-x_{i}(t)\right|<\varepsilon$. Namely $x_{i}(t)$ can be approached with any precision by polynomial function 
system $P_{k}(t) \quad(k=1,2, \cdots, K) \quad$ in $C[0, T]$, thereby $x_{i}(t)$ can be expressed as: $x_{i}(t)=\sum_{k=1}^{K} c_{i k} P_{k}(t), c_{i k} \in R$.If polynomial function system $P_{k}(t)$ is independent of each other in $C[0, T]$, then it can be orthogonalized by Gram-Schmidt orthogonalized steps, as a result, you can get a set of standard orthogonal basis functions $b_{k}(t)$ on the space of $C[0, T]$. Then $x_{i}(t)$ and $\omega_{i}(t)$ can be separately expressed as: $x_{i}(t)=\sum_{k=1}^{K} x_{i k} b_{k}(t)$, $\omega_{i}(t)=\sum_{k=1}^{K} \omega_{i k} b_{k}(t)$,among them, $x_{i k}, \omega_{i k} \in R$ is the expansion equation coefficients. According to the properties of orthogonal function, having that

$$
\int_{0}^{T} b_{k}(t) b_{l}(t) d t=\left\{\begin{array}{l}
1, k=l \\
0, k \neq l
\end{array}\right.
$$

Therefore, formula (1) can be simplified as:

$$
y=f\left(\sum_{i=1}^{n} \sum_{k=1}^{K} \omega_{i k} x_{i k}-\theta\right)
$$

\subsection{Time Series Prediction Model}

The time series prediction model that is proposed based on process neural network is a kind of three layer forward process neural network. Its input layer only has one unit, the hidden layer is formed by $\mathrm{n}$ process neurons, and output only has one unit. Model topology structure as shown in figure 2.

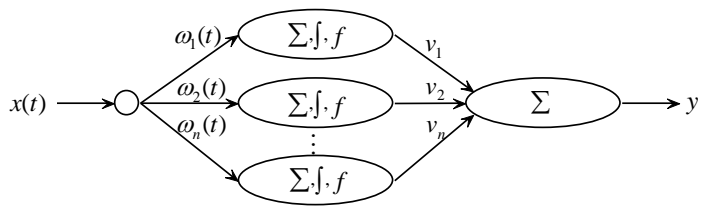

Figure. 2 Time Series Prediction Model via Process Neural Network

If $x(t)=x_{m}(t)$, then the prediction of time series short term prediction model based on process neural network for $x_{m+1}$ is:

$$
y=\sum_{i=1}^{n} v_{i} f\left(\int_{0}^{T} \omega_{i}(t) x(t) d t-\theta_{i}\right)-\theta
$$

It is knew by the foregoing that $x(t)$ can be spread as $x(t)=\sum_{k=1}^{K} a_{k} b_{k}(t), a_{k} \in R$, correspondingly $\omega_{i}(t)$ can be spread as $\omega_{i}(t)=\sum_{k=1}^{K} \omega_{i k} b_{k}(t), \omega_{i k} \in R$. Therefore, formula (3) can be simplified as:

$$
y=\sum_{i=1}^{n} v_{i} f\left(\sum_{k=1}^{K} \omega_{i k} a_{k}-\theta_{i}\right)-\theta
$$

\subsection{The Learning Algorithm}

Giving $\mathrm{L}$ set of learning sample $\left\{x_{l}(t), d_{l}\right\} \quad(l=1,2, \cdots, L), d_{l}$ is the network expected output corresponding to the sample $x_{l}(t)$, if $y_{l}$ is corresponding actual network output, then error function of network model can be defined as: 


$$
E=\frac{1}{2} \sum_{l=1}^{L}\left(y_{l}-d_{l}\right)^{2}=\frac{1}{2} \sum_{l=1}^{L}\left(\sum_{i=1}^{n} v_{i} f\left(\sum_{k=1}^{K} \omega_{i k} a_{k l}-\theta_{i}\right)-\theta-d_{l}\right)^{2}
$$

For analyzing conveniently, marking

$$
Z_{i l}=\sum_{k=1}^{K} \omega_{i k} a_{k l}-\theta_{i}
$$

According to the fast gradient descent algorithm, waiting training parameter adjustment rules of the network model is:

$$
\left\{\begin{array}{l}
v_{i}(s+1)=v_{i}(s)+\alpha \Delta v_{i}(s) \\
\omega_{i k}(s+1)=\omega_{i k}(s)+\beta \Delta \omega_{i k}(s) \\
\theta_{i}(s+1)=\theta_{i}(s)+\gamma \Delta \theta_{i}(s) \\
\theta(s+1)=\theta(s)+\lambda \Delta \theta(s)
\end{array}\right.
$$

Among them, $\alpha, \beta, \gamma, \lambda$ is the learning rate, $s$ is the learning iterations.

In each learning iteration, $\Delta v_{i} 、 \Delta \omega_{i k} 、 \Delta \theta_{i} 、 \Delta \theta$ can be separately expressed as:

$$
\left\{\begin{array}{l}
\Delta v_{i}=-\frac{\partial E}{\partial v_{i}}=-\sum_{l=1}^{L}\left(y_{l}-d_{l}\right) f\left(Z_{i l}\right) \\
\Delta \omega_{i k}=-\frac{\partial E}{\partial \omega_{i k}}=-\sum_{l=1}^{L}\left(y_{l}-d_{l}\right) v_{i} f^{\prime}\left(Z_{i l}\right) a_{k l} \\
\Delta \theta_{i}=-\frac{\partial E}{\partial \theta_{i}}=\sum_{l=1}^{L}\left(y_{l}-d_{l}\right) v_{i} f^{\prime}\left(Z_{i l}\right) \\
\Delta \theta=-\frac{\partial E}{\partial \theta}=\sum_{l=1}^{L}\left(y_{l}-d_{l}\right)
\end{array}\right.
$$

The learning process can be completely described as follows:

Step 1 spreading the input function and the connection weight function of network model by selecting the appropriate orthogonal basis function at the same time;

Step 2 giving the learning error precision $\varepsilon$; the learning iterations $s=0$, the maximum number of learning iterations $M$; the learning rate;

Step 3 initializing the waiting training parameters of the network model;

Step 4 according to the formula (5) to calculate the error function $E$, if $E<\varepsilon$ or $s>M$ then going to step6;

Step 5 adjusting the waiting training parameters according to formula (6) and (7) rules, $s+1 \rightarrow s$, going to step4;

Step 6 outputting the learning result and ending.

\section{Application and Verification}

The used data is the national monthly total power generation in January, 2001 April,2012, and it comes from the National Bureau of Statistics Database of people's Republic of China. Because the database does not contain the data of the 2005 -January, 2012 and February, 2012, thus obtained is the national power generation data for 127 months. It is regarded as equal interval sampling similarly in this text, so getting a power generation time series of containing 127 data. Constituting a time-varying function as the input of time series prediction model based on process neural network by fitting nine consecutive data in the time series, taking tenth data as the corresponding ideal output of prediction model. As a result, getting 118 groups of samples in total, and its first 80 groups of samples are used as study sample, final 38 groups of samples are used as test 
sample. Designing the topological structure of process neural network model as 1-3-1 by "heuristic method", and spreading its input function and connection weight function by the Lagrange orthogonal basis function. The learning error precision is set as 0.01 , the learning rate is $1.3 \times 10^{-3}$, the maximum number of iteration is 5000 times. The process neural network model will converge after 281 times learning iterative. In order to test the generalization ability of network model after studying, using final 38 groups of nonlearning samples to test. The test result is showed in Figure 3, the average relative error is $3.82 \%$, indicating that it is effective to predict the power generation by the time series prediction model based on process neural network.

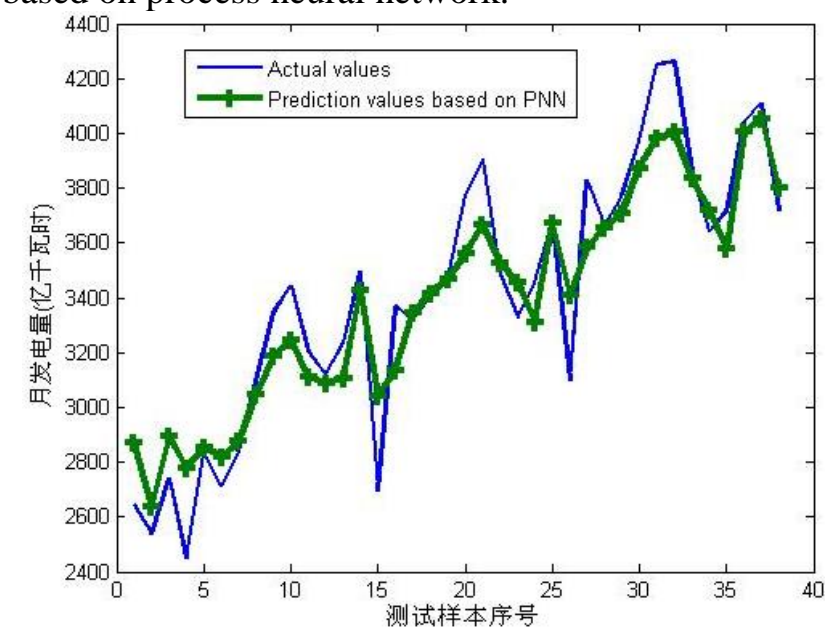

Figure 3. Power Generation Prediction based on PNN

For comparison, auto regression analysis model is used to predict the national power generation in this text. For the above time series of containing 127 power generation data, taking 9 consecutive data in the time series as the input of time series prediction model based on auto regression analysis, and taking the tenth data as the corresponding ideal output of prediction model. As a result, also getting 118 groups of samples in total, its first 80 groups of samples are used as regression analysis sample, final 38 groups of samples are used as test sample. The prediction and test result based on auto regression analysis is showed in figure 4 . The average relative error is $6.87 \%$.

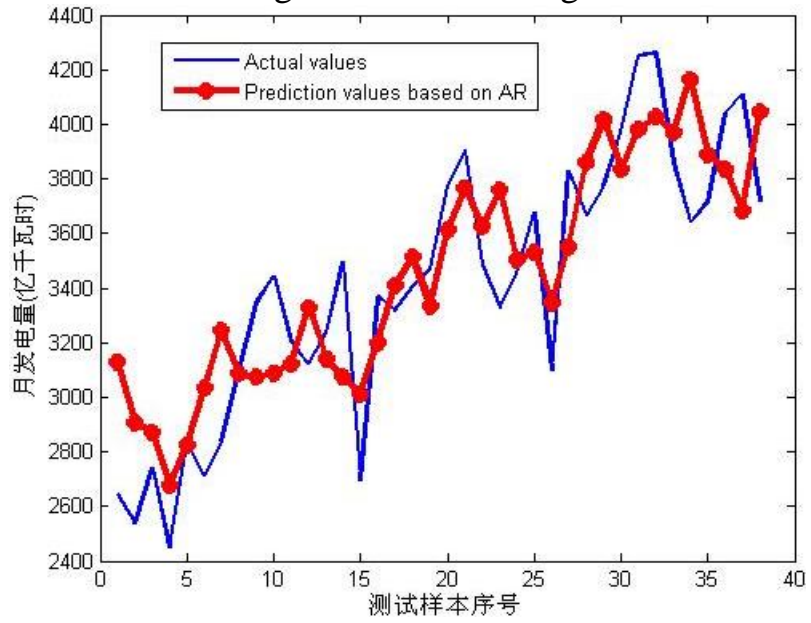

Figure 4. Power Generation Prediction based on AR 
It can be seen by comparing Figure 3 and Figure 4,because of the input of process neural network is time varying function, it can overcome the real problem that the auto regression analysis method is difficult to fully reflect the insufficiency of time cumulative effect in time series, so the prediction accuracy is relatively high.

\section{Conclusions}

In this paper, the problem of national power generation prediction is converted into a problem of time series prediction to analyze and process. Considering that the traditional time series prediction method that such as regression analysis is restricted by instantaneous synchronization of input, thus its input is a series of discrete values, so it is difficult to reflect the actual time accumulation effect in the time series, the national power generation prediction model based on process neural network is proposed. The input of proposed prediction model is time varying function, thus it can well reflect the time accumulation effect excited in time series by its integral operator, so making its higher prediction precision. The prediction model of proposed is applied to predict the national power generation in January,2001 to April ,2012, and achieving satisfactory results.

\section{Acknowledgements}

This paper is supported by University Science Park Entrepreneurial Talent of Innovation Talents of Science and Technology of Harbin Application Technology Research and Development Project (2013RFDXJ001).

\section{References}

[1] Energy Information Administration (EIA), International Energy outlook, U. S. Government, (2007), pp. 61- 71.

[2] Q. Shi and X. P. Yao, "Predicting models of power generation and installed capacity in the whole world and in China", Journal of Power Engineering, vol. 28, no. 1, (2008), pp. 147-151.

[3] D. Shao, H. Lin and Z. F. Hao, "Forecasting method of medium-term electricity consumption under the impact from the Spring Festival", Electric Machines and Control, vol. 11, no. 5, (2007), pp. 555-558.

[4] Q. Duan, J. G. Zhao and L. Niu, "Regression based on sparse Bayesian learning and the applications in electric systems", Fourth International Conference on Natural Computing, (2008); Jinan, China.

[5] J. C. Lu, S. Y. Zhang and D. X. Niu, "Power generation forecasting based on ARIMA model", Journal of North China Electric Power University, vol. 31, no. 3, (2004), pp. 78-80.

[6] Q. Duan, J. G. Zhao and Y. Ma, "Relevance vector machine based on particle swarm optimization of compounding kernels in electricity load forecasting", Electric Machines and Control, vol. 14, no. 6, (2010), pp. 33-38.

[7] G. Ding, X. Y. Fu and S. S. Zhong, "Aeroengine performance parameters prediction based on process neural network", Computer Integrated Manufacturing Systems, vol. 17, no. 1, (2011), pp. 198-207.

[8] G. Ding, L. Lin and S. S. Zhong, "Functional time series prediction using process neural network", Chinese Physics Letters, vol. 26, no. 9, (2009), pp. 090502-1 090502-4.

[9] L. Ge and G. S. Yin, "Time series clustering analysis based on wavelet and process neural networks", Electric Machines and Control, vol. 15, no. 12, (2011), pp. 78-82.

[10] X. G. He and J. Z. Liang, "Some Theoretical issues on procedure neural networks", Engineering Science, vol. 2 , no. 12 , (2000), pp. 40-44.

[11] G. Ding and S. S. Zhong, "Time series prediction based on process neural network and its application", Control and Decision, vol. 21, no. 9, (2006), pp. 1037-1041.

[12] L. I. Zhang, H. W. Tao and C. E. Holt, "A critical window for cooperation and competition among developing retinotectal synapses", Nature, vol. 395, no. 6697, (1998), pp.37-44.

[13] S. H. Xu and X. G. He, "Learning algorithms of process neural networks based on orthogonal function basis expansion", Chinese Journal of Computers, vol. 7, no. 5, (2004), pp. 645-650. 